\title{
Optimal Annuitization, Uncertain Survival Probabilities and Maxmin Preferences
}

\author{
Hippolyte d'ALBIS* \\ Paris School of Economics (University Paris 1) \\ Emmanuel THIBAULT \\ Toulouse School of Economics (University of Perpignan, CDED and IDEI)
}

\begin{abstract}
We consider a life-cycle model with bequest motives and assume that the individual does not know her survival probability and has maxmin utility preferences; we show it is optimal not to annuitize but to purchase pure life insurance policies instead.
\end{abstract}

Keywords: Demand for Annuities, Uncertain Survival Probabilities, Uncertainty Aversion, Maxmin.

JEL codes: D11, D81, G11, G22.

${ }^{*}$ Corresponding Author. H. d'Albis, CES, 106 boulevard de l'Hopital, 75013 Paris, France. Phone: 331440781 99. Fax: 331440782 31. Email: dalbis@univ-paris1.fr 


\section{Introduction}

Only a small fraction of people opt for voluntary annuitization. Such observations are at odds with the life-cycle model of consumption with uncertain lifetime proposed by Yaari (1965). Full annuitization should indeed be the optimal strategy followed by an individual without altruistic motives provided that annuities are available. The initial framework has hence been extended and many partial explanations of the socalled annuity market participation puzzle have been proposed. However, as shown by Davidoff et al. (2005), positive annuitization still remains optimal under very general specifications and assumptions, including intergenerational altruism. In this paper, we study a life-cycle model similar to that of Yaari (1965) and Davidoff et al. (2005), while restricting ourselves to the case of warm-glow altruism. We consider a maxmin utility problem within a framework with state-dependent utilities yielded by uncertain lifetimes. In this simple framework, we show it is optimal not to annuitize, even for large returns of annuities and a low degree of intergenerational altruism.

\section{Basic Framework}

We consider a two-period model of consumption and bequest with uncertain lifetime similar to that of Davidoff et al. (2005). The length of life spans two periods at the most, with the second one being uncertain. The individual derives utility from consumption and a bequest that she might leave at the end of period 1 or 2 . In the first period, the individual is endowed with a non negative initial income $\Omega$ that can be shared between bonds and annuities. Bonds return $R_{B}>0$ units of consumption in period 2, whether the individual is alive or not, in exchange for each unit of the initial endowment. Conversely, annuities return $R_{A}>R_{B}$ in period 2 if the individual is alive and nothing otherwise. Due to the possibility of dying, the demand for bonds should be non-negative and therefore annuities are the only way to borrow (this selling of annuities is a purchase of pure life insurance policies). If the individual is alive during the second period, she may allocate her financial wealth between consumption and bequest. Since death is certain at the end of period 2, the latter corresponds exclusively to a demand for bonds.

Let $c_{1}, A$ and $B$ respectively denote the consumption, the demand for annuities and the demand for bonds in the first period, and let $c_{2}$ and $x$ respectively denote the consumption and the bequest decided upon in the second period. The budget 
constraints can be written as follows:

$$
\begin{aligned}
c_{1} & =\Omega-A-B, \\
c_{2} & =R_{A} A+R_{B} B-x .
\end{aligned}
$$

In addition, the following inequalities hold:

$$
c_{1} \geq 0, c_{2} \geq 0, x \geq 0, B \geq 0 .
$$

Using the budget constraints, these inequalities imply that the demand for annuities is bounded and may be negative:

$$
-\frac{R_{B}}{R_{A}-R_{B}} \Omega \leq A \leq \Omega .
$$

In accordance with Davidoff et al. (2005), we assume that whatever the length of the individual's life, bequests are received in period 3, involving additional interest: the bequest is therefore $R_{B} x$ if the individual is alive in period 2, while it is $R_{B}^{2} B$ if she is not.

Finally, the individual's utility is $u\left(c_{1}\right)+w\left(c_{2}\right)+v\left(R_{B} x\right)$ if she lives for two periods and $u\left(c_{1}\right)+v\left(R_{B}^{2} B\right)$ if she lives for only one period. Functions $u(),. w($.$) and v($. are supposed to be positive, increasing, concave and to possess an infinite slope at zero and a horizontal slope at infinity. Additive separability is not a key assumption and our results still hold with more general functions.

\section{Optimal Annuitization and Maxmin Utility}

In what follows, the individual is assumed to be in complete ignorance about her survival probability, which may take any value between 0 and 1 . Moreover, she employs the Wald (1950) decision criterion, the so-called maxmin utility criterion. She behaves so as to maximize utility from among the worst possible utilities. In our case, there are two possible states of nature, namely being alive or not during the second period, but since utility increases with a bequest, the worst state is not given a priori. The individual hence solves:

$$
\max _{c_{1}, c_{2}, x, B} \min \left(u\left(c_{1}\right)+w\left(c_{2}\right)+v\left(R_{B} x\right), u\left(c_{1}\right)+v\left(R_{B}^{2} B\right)\right)
$$

subject to (1), (2) and (3).

Let $\left(c_{1}^{*}, c_{2}^{*}, x^{*}, A^{*}, B^{*}\right)$ be the optimal solution of the problem. The following two propositions characterize this solution. 


\section{Proposition 1 .}

The optimal behavior is to make welfare independent of the length of life by equalizing the utilities derived in the two states of nature, which implies: $w\left(c_{2}^{*}\right)+v\left(R_{B} x^{*}\right)=$ $v\left(R_{B}^{2} B^{*}\right)$. Consequently, the second-period consumption is greater than annuitization: $c_{2}^{*}>R_{A} A^{*}$.

\section{Proof - See Appendix A.}

The presence of a bequest motive implies that the utility obtained if death occurs at the end of the first period is not systematically lower than if it occurs one period later. By modifying the relative share of bonds and annuities in the portfolio, both cases are, in actual fact, possible. In Proposition 1, we suggest that the solution of the maxmin problem is to make welfare independent of the lottery i.e., in our case, independent of the length of life. The intuition for this result is explained as follows. Suppose, by contradiction, that utility is greater if the individual lives for two periods. The individual then aims to maximize the utility obtained in the event of early death, which is achieved by selling the highest possible, indeed an infinite number of annuities in order to purchase a maximum number of bonds. In this case, however, the utility in the event of early death is infinite and greater than in the event of late death. Suppose, conversely, that the utility is greater if the individual lives for one period. The individual then aims to maximize the utility obtained in the event of late death. Since annuities return more than bonds, it would therefore be optimal not to hold bonds in her portfolio. The bequest in the event of early death is therefore zero, and the utility with one period of life cannot be greater than with two periods.

To summarize, with a maxmin utility criterion, the optimal behavior is to avoid the lottery by ensuring the same utility whatever the length of life. As a consequence, the utility derived from both consumption and bequest, obtained in the event of survival, should be made equal to the utility derived from the bequest only in the event of early death. The bequest in the latter case should then be larger than in the former, which, using the budget constraint (2), implies that second-period consumption exceeds annuitization. Contrary to the expected utility case studied by Yaari (1965), the individual consumes some of her bonds if she survives and thus, a zero annuitization strategy is possible. We note that this result is true for any returns on annuities and bonds provided that $R_{A}>R_{B}$.

\section{Proposition 2 .}

The optimal strategy is to sell annuities short: $A^{*}<0$. 
Proof - See Appendix B.

With a maxmin utility criterion, the individual should not purchase annuities but sell them short, or, equivalently, purchase pure life insurance policies (Bernheim, 1991). Proposition 2 claims that the selling of annuities is the only way to sufficiently reduce consumption and bequest in the second period in order to equalize utilities in the two states of nature. To understand this result, let us figure out what would happen if the utility were independent of consumption. Equalizing the utilities in the two states of nature would then be obtained by equalizing the bequests. Since annuities return more than bonds, the optimal portfolio should be exclusively composed of bonds: the demand for annuities would thus be zero. Our model is more complex as utilities are state-dependent. If the individual is alive in the second period, she benefits from her consumption, which should be compensated by a higher utility if she were not alive; this is achieved through the selling of annuities.

Our framework can be considered as an extreme case when dealing with the demand for annuities. In the next section, we extend our results to a more general case for which the values that may be taken by the unknown survival probability do not necessarily include 0 and 1 .

\section{Extension to Maxmin Expected Utility}

In this section, we assume that the individual is still unaware of both her survival probability and its distribution but that she does know the support of the latter. More precisely, the survival probability, denoted by $p$, may take any value within the interval $\left[p_{0}, p_{1}\right]$, with $0 \leq p_{0}<p_{1} \leq 1$. For each value of $p$, an expected utility may then be computed. We assume that the individual employs a maxmin expected utility criterion (Gilboa and Schmeidler, 1989). Her problem can be written as follows:

$$
\max _{c_{1}, c_{2}, x, B} \min _{p} u\left(c_{1}\right)+p\left[w\left(c_{2}\right)+v\left(R_{B} x\right)\right]+(1-p) v\left(R_{B}^{2} B\right),
$$

subject to the same constraints as in the previous section, namely (1), (2) and (3).

We note that the problem studied in the previous section is a special case of this one. Moreover, in order to equalize the expected utilities in each state of nature (i.e. for each possible value of the survival probability) it is necessary to make the welfare independent of longevity, as stated in Proposition 1. In this case, Proposition 2 holds. In the following proposition, we give a sufficient condition under which such a behavior is still optimal. 


\section{Proposition 3 .}

There exists $\hat{p}_{0} \in\left(0, R_{B} / R_{A}\right)$, such that if $p_{0}<\hat{p}_{0}$, the optimal strategy is to sell annuities short: $A^{*}<0$.

Proof - See Appendix C.

The main result we obtain in Proposition 2 is hence extended to a maxmin expected utility framework provided that the lowest possible value of the uncertain survival probability is not too large. If $p_{0}$ is sufficiently low, the individual sells annuities short. Conversely, if $p_{0}$ is high enough, the worst state of nature may be the expected utility computed at $p_{0}$. Then, maximizing the latter objective may lead to a positive demand for annuities.

Let us point out that the annuitization decision is made by the elderly who face relatively low average survival probabilities and, consequently, even lower $p_{0}$. At first glance, the condition $p_{0}<\hat{p}_{0}$ is thus realistic.

\section{Conclusion}

In this article, we have provided a simple framework in which rational individuals do not annuitize. More precisely, we show that the optimal strategy is to sell annuities short, or to purchase pure life insurance policies. Our result hinges on two crucial assumptions, namely that individuals are unaware of their own survival probability and that they display aversion toward this uncertainty, and on one condition, which is that the lowest possible survival probability should be low enough. We have proved our result using a maxmin expected utility framework, which features a rather extreme case in terms of aversion to uncertainty. We conjecture that our results hold for sufficiently strong aversion to uncertainty. It would nevertheless be interesting to study this last point carefully.

\section{Acknowledgements}

We would like to thank Jérôme Glachant for helpful comments and suggestions. H. d'Albis thanks the Chair Dauphine-ENSAE-Groupama and E. Thibault thanks the Chair Fondation du Risque/SCOR "Marché du risque et création de valeurs" for their financial support. 


\section{References}

Bernheim, B.D, 1991. How strong are bequest motives? Evidence based on estimates of the demand for live insurance and annuities. J. Political Econ. 99, 899-927.

Davidoff, T., Brown, J.R., Diamond, P.A., 2005. Annuities and individual welfare. Am. Econ. Rev. 95, 1573-1590.

Gilboa, I., Schmeidler, D., 1989. Maxmin expected utility with non-unique prior. J. Math. Econ. 18, 141-153.

Wald, A., 1950. Statistical decision functions. Wiley, New York.

Yaari, M.E., 1965. Uncertain lifetime, life insurance, and the theory of the consumer. Rev. Econ. Stud. 32, 137-160.

\section{Appendix}

\section{Appendix A - Proof of Proposition 1.}

We first note that the objective function (5) is equivalent to the following one:

$$
\max \left(\phi\left(c_{1}, c_{2}, x, B\right), \psi\left(c_{1}, c_{2}, x, B\right)\right),
$$

where:

$$
\begin{aligned}
\phi\left(c_{1}, c_{2}, x, B\right)= & \max w\left(c_{2}\right)+v\left(R_{B} x\right) \\
& \text { s.t. } v\left(R_{B}^{2} B\right)-w\left(c_{2}\right)-v\left(R_{B} x\right) \geq 0, \\
\psi\left(c_{1}, c_{2}, x, B\right)= & \max v\left(R_{B}^{2} B\right) \\
& \text { s.t. } w\left(c_{2}\right)+v\left(R_{B} x\right)-v\left(R_{B}^{2} B\right) \geq 0 .
\end{aligned}
$$

The Lagrangians of problems (A.2) and (A.3) are respectively written as:

$$
\begin{aligned}
& \mathcal{L}_{1}=u\left(c_{1}\right)+(1-\mu)\left[w\left(c_{2}\right)+v\left(R_{B} x\right)\right]+\mu v\left(R_{B}^{2} B\right), \\
& \mathcal{L}_{2}=u\left(c_{1}\right)+\lambda\left[w\left(c_{2}\right)+v\left(R_{B} x\right)\right]+(1-\lambda) v\left(R_{B}^{2} B\right),
\end{aligned}
$$

where the multipliers associated with the inequality constraints are denoted by $\mu$ and $\lambda$. As a consequence, $\lambda=1-\mu$, and the first-order conditions of our problem are:

$$
\begin{aligned}
-u^{\prime}\left(c_{1}\right)+(1-\mu) R_{A} R_{B} v^{\prime}\left(R_{B} x\right) & =0, \\
-u^{\prime}\left(c_{1}\right)+(1-\mu) R_{B}^{2} v^{\prime}\left(R_{B} x\right)+\mu R_{B}^{2} v^{\prime}\left(R_{B}^{2} B\right) & =0, \\
(1-\mu)\left[w^{\prime}\left(c_{2}\right)-R_{B} v^{\prime}\left(R_{B} x\right)\right] & =0, \\
\mu(1-\mu)\left[w\left(c_{2}\right)+v\left(R_{B} x\right)-v\left(R_{B}^{2} B\right)\right] & =0 .
\end{aligned}
$$


We now prove the first part of Proposition 1 by showing that the multiplier $\mu$ cannot be neither zero nor one. Let us first show that $\mu \neq 0$. Using (A.6) and (A.7), $\mu=0$ is indeed impossible since $R_{A}>R_{B}$. Let us now show that $\mu \neq 1$. Using (A.6) and (A.7), $\mu=1$ would imply that $c_{1}$ and $B$ tend to $+\infty$, because it has been assumed that marginal utility is zero at infinity. Using (1), this implies that $A$ tends to $-\infty$. Then, replacing (1) in (2) gives:

$$
x+c_{2}=\left(R_{A}-R_{B}\right) A+R_{B}\left(\Omega-c_{1}\right),
$$

from which we deduce that $x+c_{2}$ tends to $-\infty$. Hence, $\mu=1$ is impossible since the inequalities in (3) cannot be satisfied.

Next, we prove the second part of Proposition 1 using (A.9) for $\mu \notin\{0,1\}$. At the optimal solution, we may write:

$$
w\left(c_{2}^{*}\right)+v\left(R_{B} x^{*}\right)=v\left(R_{B}^{2} B^{*}\right),
$$

which implies that $x^{*}<R_{B} B^{*}$. Using (2) is finally sufficient to obtain the desired conclusion.

\section{Appendix B - Proof of Proposition 2.}

Let us compare the solution of problem (6), denoted $\left(c_{1}^{*}, c_{2}^{*}, x^{*}, A^{*}, B^{*}\right)$, with the feasible set $\left(c_{1}^{*}, \bar{c}_{2}, \bar{x}, A^{*}, B^{*}\right)$ where $\bar{c}_{2}=0$ and $\bar{x} \equiv R_{A} A^{*}+R_{B} B^{*}$. Since $w^{\prime}(0)=+\infty$ is assumed, the latter does not satisfy (A.8), and is therefore not optimal. We conclude (using the assumption $w(0) \geq 0$ ) that:

$$
w\left(c_{2}^{*}\right)+v\left(R_{B} x^{*}\right)>v\left(R_{B} R_{A} A^{*}+R_{B}^{2} B^{*}\right) .
$$

Using Proposition 1, we obtain that $v\left(R_{B}^{2} B^{*}\right)>v\left(R_{B} R_{A} A^{*}+R_{B}^{2} B^{*}\right)$, and consequently that $A^{*}<0$.

\section{Appendix C - Proof of Proposition 3.}

Let us first denote the solution of (6) by $\left(c_{1}^{*}, c_{2}^{*}, x^{*}, A^{*}, B^{*}, p^{*}\right)$ and the expected utility computed at the optimum by $E U^{*}$ that we may write:

$$
E U^{*}=u\left(c_{1}^{*}\right)+p^{*}\left[w\left(c_{2}^{*}\right)+v\left(R_{B} x^{*}\right)\right]+\left(1-p^{*}\right) v\left(R_{B}^{2} B^{*}\right) .
$$

The proof proceeds as follows. We assume that $A^{*}>0$, and show that there exists a $\hat{p}_{0}$ such that, for all $p_{0} \leq \hat{p}_{0}$, this is not possible. We conclude that $A^{*}$ is negative for all $p_{0}<\hat{p}_{0}$. 
Let us consider the feasible set, denoted by $\left(\bar{c}_{1}, \bar{c}_{2}, \bar{x}, \bar{A}, \bar{B}, \bar{p}\right)$, which is such that $\bar{A} \in\left(0, A^{*}\right), \bar{p}=p^{*}, \bar{B}=B^{*}$ and $\bar{x}=R_{B} B^{*}$. We hence obtain $\bar{c}_{2}=R_{A} \bar{A}$ using $(2)$, and $\bar{c}_{1}>c_{1}^{*}$ using (1). Let $\overline{E U}$ denote the expected utility obtained by this set. Since, by the definition of $\bar{B}$ and $\bar{x}, w\left(\bar{c}_{2}\right)+v\left(R_{B} \bar{x}\right)>v\left(R_{B}^{2} \bar{B}\right)$, we have:

$$
\overline{E U}>u\left(\bar{c}_{1}\right)+v\left(R_{B}^{2} \bar{B}\right)>u\left(c_{1}^{*}\right)+v\left(R_{B}^{2} B^{*}\right) .
$$

Moreover, by the definition of a maximum: $E U^{*} \geq \overline{E U}$, from which we deduce that:

$$
E U^{*}>u\left(c_{1}^{*}\right)+v\left(R_{B}^{2} B^{*}\right) .
$$

This implies that $w\left(c_{2}^{*}\right)+v\left(R_{B} x^{*}\right)>v\left(R_{B}^{2} B^{*}\right)$ and consequently that $p^{*}=p_{0}$. Therefore, $\left(c_{1}^{*}, c_{2}^{*}, x^{*}, A^{*}, B^{*}\right)$ should be the solution of the following problem:

$$
\max _{c_{1}, c_{2}, x, B} u\left(c_{1}\right)+p_{0}\left[w\left(c_{2}\right)+v\left(R_{B} x\right)\right]+\left(1-p_{0}\right) v\left(R_{B}^{2} B\right),
$$

subject to (1), (2) and (3).

However, it can be shown that the $A$ solution of the latter problem is increasing with $p_{0}$, negative for $p_{0}=0$ and positive for $p_{0}=R_{B} / R_{A}$. Then, there exists $\hat{p}_{0} \in\left(0, R_{B} / R_{A}\right)$ such that for all $p_{0} \leq \hat{p}_{0}$, we have a contradiction between $\left(c_{1}^{*}, c_{2}^{*}, x^{*}, A^{*}, B^{*}, p^{*}\right)$ being optimal and $A^{*}>0$ being positive. We conclude that for all $p_{0}<\hat{p}_{0}$, the optimal demand is negative, i.e. $A^{*}<0$. 